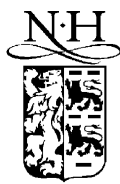

ELSEVIER
Available online at www.sciencedirect.com

science@Direct.

PHYSICS LETTERS B

Physics Letters B 568 (2003) 23-34

www.elsevier.com/locate/npe

\title{
Search for excited leptons at LEP
}

\section{L3 Collaboration}

P. Achard ${ }^{\text {t }}$, O. Adriani q, M. Aguilar-Benitez ${ }^{\mathrm{x}}$, J. Alcaraz ${ }^{\mathrm{x}}$, G. Alemanni ${ }^{\mathrm{v}}$, J. Allaby ${ }^{\mathrm{r}}$, A. Aloisio $^{\text {ab }}$, M.G. Alviggi ${ }^{\text {ab }}$, H. Anderhub ${ }^{\text {at }}$, V.P. Andreev ${ }^{\text {f,ag }}$, F. Anselmo ${ }^{\text {h }}$, A. Arefiev ${ }^{\text {aa }}$, T. Azemoon ${ }^{\text {c }}$, T. Aziz ${ }^{\text {i, }}$ P. Bagnaia ${ }^{\text {al }}$, A. Bajo ${ }^{\mathrm{x}}$, G. Baksay ${ }^{\mathrm{y}}, \mathrm{L}$. Baksay $^{\mathrm{y}}$, S.V. Baldew ${ }^{\text {b }}$, S. Banerjee ${ }^{\text {i }}$, Sw. Banerjee ${ }^{\mathrm{d}}$, A. Barczyk ${ }^{\text {at,ar }}$, R. Barillère ${ }^{\mathrm{r}}$, P. Bartalini ${ }^{\mathrm{v}}$, M. Basile ${ }^{\mathrm{h}}$, N. Batalova ${ }^{\mathrm{aq}}, \mathrm{R}$. Battiston ${ }^{\text {af }}$, A. Bay ${ }^{\mathrm{v}}$, F. Becattini ${ }^{\mathrm{q}}, \mathrm{U}^{\mathrm{f}}$ Becker ${ }^{\mathrm{m}}$, F. Behner ${ }^{\text {at }}$, L. Bellucci ${ }^{q}$, R. Berbeco ${ }^{c}$, J. Berdugo $^{\mathrm{x}}$, P. Berges $^{\mathrm{m}}$, B. Bertucci ${ }^{\text {af }}$, B.L. Betev ${ }^{\text {at }}$, M. Biasini ${ }^{\text {af }}$, M. Biglietti ${ }^{\text {ab }}$, A. Biland ${ }^{\text {at }}$, J.J. Blaising ${ }^{\text {d }}$, S.C. Blyth ${ }^{\text {ah }}$, G.J. Bobbink ${ }^{\text {b }}$, A. Böhm ${ }^{\text {a }}$, L. Boldizsar ${ }^{1}$, B. Borgia ${ }^{\text {al }}$, S. Bottai ${ }^{\mathrm{q}}$, D. Bourilkov ${ }^{\text {at }}$, M. Bourquin ${ }^{t}$, S. Braccini ${ }^{\text {t, J.G. Branson }}{ }^{\text {an }}$, F. Brochu ${ }^{\text {d }}$, J.D. Burger ${ }^{\mathrm{m}}$, W.J. Burger ${ }^{\text {af }}$, X.D. Cai ${ }^{\mathrm{m}}$, M. Capell ${ }^{\mathrm{m}}$, G. Cara Romeo ${ }^{\mathrm{h}}$, G. Carlino ${ }^{\mathrm{ab}}$, A. Cartacci ${ }^{\mathrm{q}}$, J. Casaus ${ }^{\mathrm{x}}$, F. Cavallari ${ }^{\text {al }}$, N. Cavallo ${ }^{\text {ai }}$, C. Cecchi ${ }^{\text {af }}$, M. Cerrada ${ }^{\mathrm{x}}$, M. Chamizo ${ }^{t}$, Y.H. Chang ${ }^{\text {av }}$, M. Chemarin ${ }^{\text {w }}$, A. Chen ${ }^{\text {av }}$, G. Chen ${ }^{\mathrm{g}}$, G.M. Chen ${ }^{\mathrm{g}}$, H.F. Chen ${ }^{\mathrm{u}}$, H.S. Chen ${ }^{\mathrm{g}}$, G. Chiefari ${ }^{\mathrm{ab}}$, L. Cifarelli ${ }^{\mathrm{am}}$, F. Cindolo ${ }^{\mathrm{h}}$, I. Clare ${ }^{\mathrm{m}}$, R. Clare $^{\mathrm{ak}}$, G. Coignet $^{\mathrm{d}}$, N. Colino ${ }^{x}$, S. Costantini ${ }^{\text {al }}$, B. de la Cruz ${ }^{x}$, S. Cucciarelli af ${ }^{\text {, J.A. van Dalen }}{ }^{\text {ad }}$, R. de Asmundis ${ }^{\mathrm{ab}}$, P. Déglon ${ }^{\mathrm{t}}$, J. Debreczeni ${ }^{1}$, A. Degré ${ }^{\mathrm{d}}$, K. Dehmelt ${ }^{\mathrm{y}}$, K. Deiters ${ }^{\mathrm{ar}}$, D. della Volpe ${ }^{\text {ab }}$, E. Delmeire ${ }^{t}$, P. Denes ${ }^{\text {aj }}$, F. DeNotaristefani ${ }^{\text {al }}$, A. De Salvo ${ }^{\text {at }}$, M. Diemoz ${ }^{\text {al }}$, M. Dierckxsens ${ }^{b}$, C. Dionisi ${ }^{\text {al }}$, M. Dittmar ${ }^{\text {at }}$, A. Doria ${ }^{\text {ab }}$, M.T. Dova $^{\mathrm{j}, 5}$, D. Duchesneau ${ }^{d}$, M. Duda ${ }^{\text {a }}$, B. Echenard ${ }^{\text {t }}$, A. Eline ${ }^{\mathrm{r}}$, A. El Hage ${ }^{\mathrm{a}}$, H. El Mamouni ${ }^{\text {w, }}$ A. Engler $^{\text {ah }}$, F.J. Eppling ${ }^{\mathrm{m}}$, P. Extermann ${ }^{\mathrm{t}}$, M.A. Falagan ${ }^{\mathrm{x}}$, S. Falciano ${ }^{\text {al }}$, A. Favara ${ }^{\text {ae }}$, J. Fay ${ }^{\text {w }}$, O. Fedin ${ }^{\text {ag }}$, M. Felcini ${ }^{\text {at }}$, T. Ferguson ${ }^{\text {ah }}$, H. Fesefeldt ${ }^{\text {a }}$, E. Fiandrini ${ }^{\text {af }}$, J.H. Field $^{\mathrm{t}}$, F. Filthaut ${ }^{\text {ad }}$, P.H. Fisher ${ }^{\mathrm{m}}$, W. Fisher ${ }^{\text {aj }}$, I. Fisk ${ }^{\text {an }}$, G. Forconi ${ }^{\mathrm{m}}$, K. Freudenreich ${ }^{\text {at }}$, C. Furetta ${ }^{z}$, Yu. Galaktionov ${ }^{\text {aa, }}$, S.N. Ganguli ${ }^{i}$, P. Garcia-Abia ${ }^{\mathrm{x}}$, M. Gataullin ${ }^{\text {ae }}$, S. Gentile al, S. Giagu ${ }^{\text {al }}$, Z.F. Gong ${ }^{\text {a }}$, G. Grenier ${ }^{\mathrm{w}}$, O. Grimm ${ }^{\text {at }}$, M.W. Gruenewald ${ }^{p}$, M. Guida ${ }^{\text {am }}$, R. van Gulik ${ }^{\mathrm{b}}$, V.K. Gupta ${ }^{\mathrm{aj}}$, A. Gurtu ${ }^{\mathrm{i}}$, L.J. Gutay ${ }^{\mathrm{aq}}$, D. Haas ${ }^{\mathrm{e}}$, R.Sh. Hakobyan ${ }^{\text {ad }}$, J.M. Hansen ${ }^{\mathrm{r}}$, D. Hatzifotiadou ${ }^{\mathrm{h}}$, T. Hebbeker ${ }^{\mathrm{a}}$, A. Hervé ${ }^{\mathrm{r}}$, J. Hirschfelder ${ }^{\text {ah }}$, H. Hofer ${ }^{\text {at }}$, M. Hohlmann ${ }^{y}$, G. Holzner ${ }^{\text {at }}$, S.R. Hou ${ }^{\text {av }}$, Y. Hu ${ }^{\text {ad }}$, B.N. Jin ${ }^{\mathrm{g}}$, L.W. Jones ${ }^{\mathrm{c}}$, P. de Jong ${ }^{\mathrm{b}}$, I. Josa-Mutuberría ${ }^{\mathrm{x}}$, D. Käfer ${ }^{\mathrm{a}}$, M. Kaur ${ }^{\mathrm{n}}$, M.N. Kienzle-Focacci ${ }^{\mathrm{t}}$, J.K. Kim ${ }^{\text {ap }}$, J. Kirkby ${ }^{\mathrm{r}}$, W. Kittel ${ }^{\text {ad }}$, A. Klimentov ${ }^{\text {m,aa }}$, A.C. König ${ }^{\text {ad }}$, M. Kopal ${ }^{\text {aq }}$, V. Koutsenko ${ }^{\text {m,aa }}$, M. Kräber ${ }^{\text {at }}$, 
R.W. Kraemer ${ }^{\text {ah }}$, A. Krüger ${ }^{\text {as }}$, A. Kunin ${ }^{\mathrm{m}}$, P. Ladron de Guevara ${ }^{\mathrm{x}}$, I. Laktineh ${ }^{\mathrm{w}}$, G. Landi ${ }^{q}$, M. Lebeau ${ }^{\mathrm{r}}$, A. Lebedev ${ }^{\mathrm{m}}$, P. Lebrun ${ }^{\mathrm{w}}$, P. Lecomte ${ }^{\text {at }}$, P. Lecoq $^{\mathrm{r}}$, P. Le Coultre ${ }^{\text {at }}$, J.M. Le Goff ${ }^{\mathrm{r}}$, R. Leiste ${ }^{\text {as }}$, M. Levtchenko ${ }^{\mathrm{z}}$, P. Levtchenko ${ }^{\text {ag }}, \mathrm{C}^{\mathrm{C}} \mathrm{Li}^{\text {u }}$, S. Likhoded ${ }^{\text {as }}$, C.H. Lin ${ }^{\text {av }}$, W.T. Lin ${ }^{\text {av }}$, F.L. Linde ${ }^{b}$, L. Lista ${ }^{\text {ab }}$, Z.A. Liu ${ }^{g}$, W. Lohmann ${ }^{\text {as }}$, E. Longo ${ }^{\text {al }}$, Y.S. Lu ${ }^{\mathrm{g}}$, C. Luci ${ }^{\text {al }}$, L. Luminari ${ }^{\text {al }}$, W. Lustermann ${ }^{\text {at }}$, W.G. Ma ${ }^{\text {u }}$ L. Malgeri ${ }^{\mathrm{t}}$, A. Malinin ${ }^{\text {aa }}$, C. Maña ${ }^{\mathrm{x}}$, J. Mans ${ }^{\text {aj }}$, J.P. Martin ${ }^{\mathrm{w}}$, F. Marzano al, K. Mazumdar ${ }^{\mathrm{i}}$, R.R. McNeil ${ }^{\mathrm{f}}$, S. Mele ${ }^{\mathrm{r}, \mathrm{ab}}$, L. Merola ${ }^{\mathrm{ab}}$, M. Meschini ${ }^{\mathrm{q}}$, W.J. Metzger ${ }^{\text {ad }}$, A. Mihul ${ }^{\text {}}$, H. Milcent ${ }^{\mathrm{r}}$, G. Mirabelli al ${ }^{\text {, J. Mnich }}{ }^{\mathrm{a}}$, G.B. Mohanty ${ }^{\mathrm{i}}$, G.S. Muanza ${ }^{\mathrm{w}}$, A.J.M. Muijs ${ }^{\text {b }}$, B. Musicar ${ }^{\text {an }}$, M. Musy ${ }^{\text {al }}$, S. Nagy ${ }^{\circ}$, S. Natale ${ }^{t}$, M. Napolitano ${ }^{\text {ab }}$, F. Nessi-Tedaldi at ${ }^{\text {at } H . ~ N e w m a n ~}{ }^{\text {ae }}$, A. Nisati ${ }^{\text {al }}$, H. Nowak ${ }^{\text {as }}$, R. Ofierzynski ${ }^{\text {at }}$, G. Organtini ${ }^{\text {al }}$, I. Pal ${ }^{\text {aq }}$, C. Palomares ${ }^{\mathrm{x}}$, P. Paolucci ${ }^{\text {ab }}$, R. Paramatti ${ }^{\text {al }}$, G. Passaleva ${ }^{\text {q }}$, S. Patricelli ${ }^{\text {ab }}$, T. Paul ${ }^{j}$, M. Pauluzzi ${ }^{\text {af }}$, C. Paus ${ }^{\mathrm{m}}$, F. Pauss ${ }^{\text {at }}$, M. Pedace ${ }^{\text {al }}$, S. Pensotti ${ }^{z}$, D. Perret-Gallix ${ }^{d}$, B. Petersen ${ }^{\text {ad }}$, D. Piccolo ${ }^{\text {ab }}$, F. Pierella ${ }^{\text {h}}$, M. Pioppi ${ }^{\text {af }}$, P.A. Piroué aj, E. Pistolesi $^{\text {z }}$, V. Plyaskin ${ }^{\text {aa }}$, M. Pohl ${ }^{\text {t }}$, V. Pojidaev ${ }^{\mathrm{q}}$, J. Pothier $^{\mathrm{r}}$, D. Prokofiev ${ }^{\mathrm{ag}}$, J. Quartieri ${ }^{\text {am }}$, G. Rahal-Callot ${ }^{\text {at }}$, M.A. Rahaman ${ }^{\mathrm{i}}$, P. Raics ${ }^{\circ}$, N. Raja ${ }^{\mathrm{i}}$, R. Ramelli ${ }^{\text {at }}$, P.G. Rancoita ${ }^{\text {z }}$, R. Ranieri ${ }^{\mathrm{q}}$, A. Raspereza ${ }^{\text {as }}$, P. Razis ${ }^{\text {ac }}$, D. Ren ${ }^{\text {at }}$, M. Rescigno ${ }^{\text {al }}$, S. Reucroft ${ }^{j}$, S. Riemann ${ }^{\text {as }}$, K. Riles ${ }^{\text {c }}$, B.P. Roe ${ }^{c}$, L. Romero $^{x}$, A. Rosca ${ }^{\text {as }}$, S. Rosier-Lees ${ }^{d}$, S. Roth ${ }^{a}$, C. Rosenbleck ${ }^{a}$, J.A. Rubio ${ }^{r}$, G. Ruggiero ${ }^{q}$, H. Rykaczewski ${ }^{\text {at }}$, A. Sakharov ${ }^{\text {at }}$, S. Saremi ${ }^{\text {f }}$, S. Sarkar ${ }^{\text {al }}$, J. Salicio ${ }^{\text {r }}$, E. Sanchez ${ }^{\mathrm{x}}$, C. Schäfer ${ }^{r}$, V. Schegelsky ${ }^{\text {ag }}$, H. Schopper ${ }^{\text {au }}$, D.J. Schotanus ${ }^{\text {ad }}$, C. Sciacca ${ }^{\text {ab }}$, L. Servoli ${ }^{\text {af }}$, S. Shevchenko ${ }^{\text {ae }}$, N. Shivarov ${ }^{\text {ao }}$, V. Shoutko ${ }^{\mathrm{m}}$, E. Shumilov ${ }^{\text {aa }}$, A. Shvorob ${ }^{\text {ae }}$, D. Son ${ }^{\text {ap }}$, C. Souga ${ }^{\text {w }}$, P. Spillantini ${ }^{\text {q }}$, M. Steuer ${ }^{\mathrm{m}}$, D.P. Stickland ${ }^{\text {aj }}$, B. Stoyanov ${ }^{\text {ao }}$, A. Straessner ${ }^{\text {r }}$ K. Sudhakar ${ }^{\text {i }}$, G. Sultanov ${ }^{\text {ao }}$, L.Z. Sun ${ }^{\text {u }}$, S. Sushkov ${ }^{\text {a }}$, H. Suter ${ }^{\text {at }}$ J.D. Swain ${ }^{\mathrm{j}}$, Z. Szillasi ${ }^{\mathrm{y}, 3}$, X.W. Tang ${ }^{\mathrm{g}}$, P. Tarjan ${ }^{\mathrm{o}}$, L. Tauscher ${ }^{\mathrm{e}}$, L. Taylor ${ }^{\mathrm{j}}$, B. Tellili ${ }^{\mathrm{w}}$, D. Teyssier ${ }^{\mathrm{w}}$, C. Timmermans ${ }^{\mathrm{ad}}$, Samuel C.C. Ting ${ }^{\mathrm{m}}$, S.M. Ting ${ }^{\mathrm{m}}$, S.C. Tonwar ${ }^{\mathrm{i}}$, J. Tóth ${ }^{1}$, C. Tully ${ }^{\text {aj }}$, K.L. Tung ${ }^{\mathrm{g}}$, J. Ulbricht ${ }^{\text {at }}$, E. Valente ${ }^{\text {al }}$, R.T. Van de Walle ${ }^{\text {ad }}$, R. Vasquez ${ }^{\text {aq }}$, V. Veszpremi ${ }^{y}$, G. Vesztergombi ${ }^{1}$, I. Vetlitsky ${ }^{\text {aa }}$, D. Vicinanza ${ }^{\text {am }}$, G. Viertel ${ }^{\text {at }}$, S. Villa ${ }^{\text {ak }}$, M. Vivargent ${ }^{\mathrm{d}}$, S. Vlachos ${ }^{\mathrm{e}}$, I. Vodopianov ${ }^{\mathrm{y}}$, H. Vogel ${ }^{\text {ah }}$, H. Vogt ${ }^{\text {as }}$, I. Vorobiev ${ }^{\text {ah,aa }}$, A.A. Vorobyov ${ }^{\text {ag }}$, M. Wadhwa ${ }^{\text {e }}$, Q. Wang ${ }^{\text {ad }}$, X.L. Wang " ${ }^{\text {, Z.M. Wang }}{ }^{\mathrm{u}}$, M. Weber ${ }^{\mathrm{a}}$, P. Wienemann ${ }^{\mathrm{a}}$, H. Wilkens ${ }^{\text {ad }}$, S. Wynhoff ${ }^{\text {aj }}$, L. Xia $^{\text {ae }}$, Z.Z. Xu ${ }^{\text {u }}$, J. Yamamoto ${ }^{\text {c }}$, B.Z. Yang ${ }^{\text {u }}$, C.G. Yang ${ }^{\mathrm{g}}$, H.J. Yang ${ }^{\mathrm{c}}$, M. Yang ${ }^{\mathrm{g}}$, S.C. Yeh ${ }^{\text {aw }}$, An. Zalite ${ }^{\mathrm{ag}}$, Yu. Zalite ${ }^{\mathrm{ag}}$, Z.P. Zhang ${ }^{\mathrm{u}}$, J. Zhao ${ }^{\mathrm{u}}$, G.Y. Zhu ${ }^{\mathrm{g}}$, R.Y. Zhu ${ }^{\text {ae }}$, H.L. Zhuang ${ }^{\mathrm{g}}$, A. Zichichi ${ }^{\text {h,r,s }}$, B. Zimmermann ${ }^{\text {at }}$, M. Zöller ${ }^{\text {a }}$

a III. Physikalisches Institut, RWTH, D-52056 Aachen, Germany ${ }^{1}$

${ }^{\mathrm{b}}$ National Institute for High Energy Physics, NIKHEF, and University of Amsterdam, NL-1009 DB Amsterdam, The Netherlands c University of Michigan, Ann Arbor, MI 48109, USA

d Laboratoire d'Annecy-le-Vieux de Physique des Particules, LAPP, IN2P3-CNRS, BP 110, F-74941 Annecy-le-Vieux Cedex, France e Institute of Physics, University of Basel, CH-4056 Basel, Switzerland

${ }^{f}$ Louisiana State University, Baton Rouge, LA 70803, USA

$\mathrm{g}$ Institute of High Energy Physics, IHEP, 100039 Beijing, China 6 


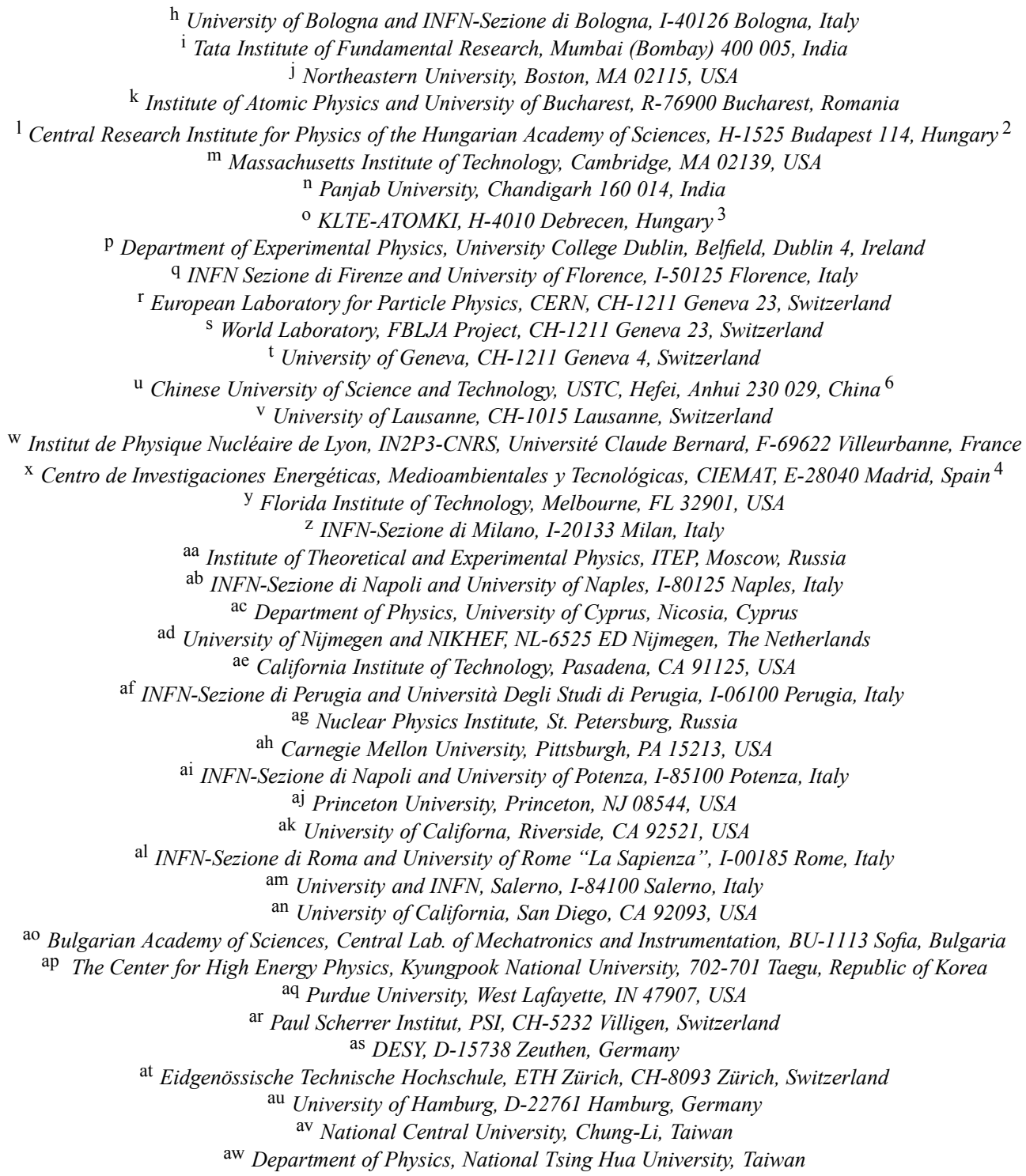

Received 26 March 2003; received in revised form 20 May 2003; accepted 27 May 2003

Editor: L. Rolandi

\begin{abstract}
A search for charged and neutral excited leptons is performed in $217 \mathrm{pb}^{-1}$ of $\mathrm{e}^{+} \mathrm{e}^{-}$collision data collected with the L3 detector at LEP at centre-of-mass energies from 202 up to $209 \mathrm{GeV}$. The pair- and single-production mechanisms of excited electrons, muons and taus, as well as of excited electron-, muon- and tau-neutrinos, are investigated and no signals are detected. Combining with L3 results from searches at lower centre-of-mass energies, gives improved limits on the masses and couplings of excited leptons.
\end{abstract}


(C) 2003 Published by Elsevier B.V.

\section{Introduction}

Charged $\left(\mathrm{e}^{*}, \mu^{*}\right.$, and $\left.\tau^{*}\right)$ and neutral $\left(v_{\mathrm{e}}^{*}, v_{\mu}^{*}\right.$ and $v_{\tau}^{*}$ ) excited leptons are predicted by composite models where leptons and quarks have substructure [1-3]. These models address fundamental questions left open by the Standard Model [4], such as the number of families and the fermion mass values.

High energy electron-positron annihilations constitute an excellent environment for the search for excited leptons and several searches have been carried out at LEP [5-7]. Searches for excited electrons and neutrinos were also performed at the HERA [8] ep collider. This Letter describes the extension of previous L3 searches $[5,6]$ to the highest centre-of-mass energies, $\sqrt{s}$, attained by the LEP machine in its last year of operation, $\sqrt{s}=202-209 \mathrm{GeV}$.

Excited leptons are studied within a model [1] in which they are described as isospin doublets with leftand right-handed components:

$\mathrm{L}^{*}=\left(\begin{array}{c}\nu^{*} \\ \ell^{*}\end{array}\right)_{L}+\left(\begin{array}{c}\nu^{*} \\ \ell^{*}\end{array}\right)_{R}$,

with $\ell=\mathrm{e}, \mu, \tau$ and $v=v_{\mathrm{e}}, v_{\mu}, v_{\tau}$. These excited leptons are accessible at LEP through pair production, $\mathrm{e}^{+} \mathrm{e}^{-} \rightarrow \ell^{*} \ell^{*}, v^{*} v^{*}$, or single production, $\mathrm{e}^{+} \mathrm{e}^{-} \rightarrow$ $\ell \ell^{*}, v v^{*}$. Pair-production searches are sensitive to excited leptons of mass up to values close to the kinematic limit $\sqrt{s} / 2$, while the single-production mechanism extends the search potential up to masses close to $\sqrt{s}$.

\footnotetext{
${ }^{1}$ Supported by the German Bundesministerium für Bildung, Wissenschaft, Forschung und Technologie.

2 Supported by the Hungarian OTKA fund under contract numbers T019181, F023259 and T037350.

3 Also supported by the Hungarian OTKA fund under contract number T026178.

${ }^{4}$ Supported also by the Comisión Interministerial de Ciencia y Tecnología.

5 Also supported by CONICET and Universidad Nacional de La Plata, CC 67, 1900 La Plata, Argentina.

6 Supported by the National Natural Science Foundation of China.
}

The pair production of excited leptons is described by the effective Lagrangian:

$\mathcal{L}_{\mathrm{L}^{*} \mathrm{~L}^{*}}=\overline{\mathrm{L}}^{*} \gamma^{\mu}\left(g \frac{\vec{\tau}}{2} \vec{W}_{\mu}+g^{\prime} Y B_{\mu}\right) \mathrm{L}^{*}$

while the single production of excited leptons and their decay into Standard Model leptons is modeled by:

$$
\begin{aligned}
\mathcal{L}_{\mathrm{L}^{*} \mathrm{~L}}= & \overline{\mathrm{L}}^{*} \sigma^{\mu \nu}\left(g \frac{f}{\Lambda} \frac{\vec{\tau}}{2} \partial_{\mu} \vec{W}_{\nu}+g^{\prime} \frac{f^{\prime}}{\Lambda} Y \partial_{\mu} B_{\nu}\right) \frac{1-\gamma^{5}}{2} \mathrm{~L} \\
& + \text { (hermitian conjugate) }
\end{aligned}
$$

In these expressions, $\gamma^{\mu}$ are the Dirac matrices, $\sigma^{\mu \nu}=$ $i\left[\gamma^{\mu}, \gamma^{\nu}\right] / 2, g$ and $g^{\prime}$ are the Standard Model SU(2) and $\mathrm{U}(1)$ coupling constants, $\vec{\tau}$ denotes the Pauli matrices, $Y=-1 / 2$ is the hypercharge and $\vec{W}$ and $B$ are the gauge fields associated with the $\mathrm{SU}(2)$ and $\mathrm{U}(1)$ groups, respectively. L denotes the Standard Model leptons, $\Lambda$ is the scale of the New Physics responsible for the existence of excited leptons and $f$ and $f^{\prime}$ scale the $\mathrm{SU}(2)$ and $\mathrm{U}(1)$ couplings, respectively.

The cross section for pair production of excited leptons depends only on their mass and on $\sqrt{s}$. As an example, for masses of the excited leptons of $101 \mathrm{GeV}$, the cross sections at $\sqrt{s}=206 \mathrm{GeV}$ for $\mathrm{e}^{+} \mathrm{e}^{-} \rightarrow \mu^{*} \mu^{*}$ and $\mathrm{e}^{+} \mathrm{e}^{-} \rightarrow v_{\mu}^{*} \nu_{\mu}^{*}$ are $0.6 \mathrm{pb}$ and $0.3 \mathrm{pb}$, respectively. The single-production cross section for excited leptons depends also (Eq. (3)) on $f / \Lambda$ and $f^{\prime} / \Lambda$.

Eq. (3) also describes the decay of excited leptons into Standard Model leptons in association with a photon or a gauge boson. Three decays are possible: radiative decays, $\ell^{*} \rightarrow \ell \gamma$ and $\nu^{*} \rightarrow v \gamma$, chargedcurrent decays, $\ell^{*} \rightarrow v \mathrm{~W}$ and $v^{*} \rightarrow \ell \mathrm{W}$, and neutralcurrent decays $\ell^{*} \rightarrow \ell Z$ and $v^{*} \rightarrow v Z$. The branching fractions in these different modes depend on the relative values of $f$ and $f^{\prime}$. As an example, Table 1 lists these fractions for two mass values and the two extreme cases $f=f^{\prime}$ and $f=-f^{\prime}$. For $f=f^{\prime}$, the radiative decay is allowed for charged excited leptons whereas it is forbidden for excited neutrinos. The opposite holds for $f=-f^{\prime}$. The final state topologies and hence the experimental sensitivity for the two scenarios are therefore very different. Table 2 
summarises all the final states for excited lepton production and decay that are considered in this Letter.

\section{Data and Monte Carlo samples}

The data sample discussed in this Letter comprises $216.9 \mathrm{pb}^{-1}$ collected with the L3 detector [9] at $\sqrt{s}=$ 202-209 GeV with an average centre-of-mass energy of $206 \mathrm{GeV}$.

Monte Carlo samples of pair-produced excited leptons are generated for a mass of $101 \mathrm{GeV}$, which is close to the expected kinematic limit. The single production of excited leptons is modeled for masses of 110,160 and $201 \mathrm{GeV}$. An interpolation, which includes samples at 100,150 and $195 \mathrm{GeV}$ produced at $\sqrt{s}=192-202 \mathrm{GeV}$ [6], allows the estimation of selection efficiencies in the mass range from 90 to $209 \mathrm{GeV}$. All possible final states listed in Table 2 are generated, including both hadronic and leptonic decays of the $\mathrm{W}$ and $\mathrm{Z}$ bosons. Differential cross sections are modeled according to Ref. [1] and initial state radiation is taken into account in cross section calculations.

Table 1

Predicted branching ratios for charged and neutral excited lepton decays, for different choices of masses $(M)$ and couplings

\begin{tabular}{lccccc}
\hline Decay channel & \multicolumn{4}{c}{ Branching ratios } \\
\cline { 2 - 3 } \cline { 2 - 3 } \cline { 5 - 6 } & \multicolumn{2}{c}{$M=102 \mathrm{GeV}$} & & \multicolumn{2}{c}{$M=200 \mathrm{GeV}$} \\
\cline { 2 - 3 } \cline { 5 - 6 }$\ell^{*} \rightarrow \ell=f^{\prime}$ & $f=-f^{\prime}$ & & $f=f^{\prime}$ & $f=-f^{\prime}$ \\
$\ell^{*} \rightarrow \nu \mathrm{W}$ & $70 \%$ & - & & $35 \%$ & - \\
$\ell^{*} \rightarrow \ell \mathrm{Z}$ & $28 \%$ & $83 \%$ & & $55 \%$ & $63 \%$ \\
$\nu^{*} \rightarrow \nu \gamma$ & $2 \%$ & $17 \%$ & & $10 \%$ & $37 \%$ \\
$\nu^{*} \rightarrow \ell \mathrm{W}$ & - & $70 \%$ & & - & $35 \%$ \\
$\nu^{*} \rightarrow \nu \mathrm{Z}$ & $83 \%$ & $28 \%$ & & $63 \%$ & $55 \%$ \\
\hline
\end{tabular}

Standard Model background processes are simulated with several Monte Carlo generators. Radiative Bhabha events are generated using BHWIDE [10] and TEEGG [11]. KK2F [12] is used for the $\mathrm{e}^{+} \mathrm{e}^{-} \rightarrow$ $\mu \mu(\gamma), \mathrm{e}^{+} \mathrm{e}^{-} \rightarrow \tau \tau(\gamma), \mathrm{e}^{+} \mathrm{e}^{-} \rightarrow \nu \nu(\gamma)$ and $\mathrm{e}^{+} \mathrm{e}^{-} \rightarrow$ $\mathrm{q} \overline{\mathrm{q}}(\gamma)$ processes. The $\mathrm{e}^{+} \mathrm{e}^{-} \rightarrow \mathrm{W}^{+} \mathrm{W}^{-}$process is modeled with KORALW [13], with the exception of the q⿱亠 $\mathrm{e} v$ final state, described by EXCALIBUR [14] which is also used for the $\mathrm{e}^{+} \mathrm{e}^{-} \rightarrow \ell \ell \ell \ell$ and $\mathrm{e}^{+} \mathrm{e}^{-} \rightarrow$ $\ell \ell \nu v$ processes. PYTHIA [15] is used for the final states coming from $\mathrm{e}^{+} \mathrm{e}^{-} \rightarrow \mathrm{ZZ}$ not covered by EXCALIBUR. GGG [16] describes the $\mathrm{e}^{+} \mathrm{e}^{-} \rightarrow \gamma \gamma(\gamma)$ process. The production of hadrons and leptons in twophoton interactions is described by PHOJET [17] and DIAG36 [18], respectively.

The L3 detector response is simulated for all Monte Carlo samples using the GEANT program [19], which includes the effects of energy loss, multiple scattering and showering in the detector. Time dependent detector behaviour, as monitored during the data taking period, is also taken into account.

\section{Selection strategy}

The search for charged and neutral excited leptons follows the one already performed at lower centre-ofmass energies $[5,6]$. Several selections are devised in order to cover all final states listed in Table 2. All those selections proceed from the identification of photons, leptons and jets and then assemble those constituents to single out the particular signature of each final state.

Photons and electrons are identified in the electromagnetic calorimeter as clusters within a polar angle with respect to the beam line which satisfies $|\cos \theta|<$ 0.95 and with energy above $1 \mathrm{GeV}$. The shape of the shower in the crystals must be compatible with a photon or an electron. Electrons must be associated to

Table 2

The final states for excited lepton production considered in this Letter, where $\ell$ runs on the lepton flavour: e, $\mu$ and $\tau$

\begin{tabular}{|c|c|c|c|c|}
\hline \multirow[t]{2}{*}{ Decay mode } & \multicolumn{2}{|c|}{ Pair production } & \multicolumn{2}{|c|}{ Single production } \\
\hline & $\mathrm{e}^{+} \mathrm{e}^{-} \rightarrow \ell^{*} \ell^{*}$ & $\mathrm{e}^{+} \mathrm{e}^{-} \rightarrow v^{*} v^{*}$ & $\mathrm{e}^{+} \mathrm{e}^{-} \rightarrow \ell \ell^{*}$ & $\mathrm{e}^{+} \mathrm{e}^{-} \rightarrow v v^{*}$ \\
\hline Radiative & $\ell^{*} \ell^{*} \rightarrow \ell \ell \gamma \gamma$ & $v^{*} v^{*} \rightarrow v v \gamma \gamma$ & $\ell \ell^{*} \rightarrow \ell \ell \gamma$ & $v v^{*} \rightarrow v v \gamma$ \\
\hline Charged-current & $\ell^{*} \ell^{*} \rightarrow \nu \nu \mathrm{WW}$ & $v_{\ell}^{*} v_{\ell}^{*} \rightarrow \ell \ell \mathrm{WW}$ & $v_{\ell} v_{\ell}^{*} \rightarrow v_{\ell} \ell \mathrm{W}$ & $\ell \ell^{*} \rightarrow \ell v_{\ell} \mathrm{W}$ \\
\hline Neutral-current & - & + & $\ell \ell^{*} \rightarrow \ell \ell Z$ & $v v^{*} \rightarrow v v Z$ \\
\hline Mixed & $\ell^{*} \ell^{*} \rightarrow \ell \gamma \nu \mathrm{W}$ & $v_{\ell}^{*} v_{\ell}^{*} \rightarrow v \gamma \ell \mathrm{W}$ & - & - \\
\hline
\end{tabular}


tracks in the central tracker, while no track is allowed near photon candidates.

Muons are selected from tracks reconstructed in the central or forward-backward muon spectrometers. These tracks must point to the interaction vertex. Signals in the time-of-flight system are used to reject background from cosmic rays. In addition, muon candidates without a track in the spectrometer are also built starting from energy depositions in the electromagnetic and hadronic calorimeters consistent with a minimum ionising particle matched to a track in the central tracker.

Tau leptons are reconstructed from low multiplicity narrow hadronic jets, or identified through their decay into electrons or muons with reconstructed missing energy $(\not \notin)$ and momentum.

Jets are reconstructed from charged tracks and energy clusters in the electromagnetic and hadronic calorimeters. The missing energy and missing momentum of the event, used to tag events with neutrinos in the final state, are calculated from all tracks, energy clusters and additional muons. The missing momentum is required to point away from the beam axis in most of the selections, so as to minimise the background from two-photon interactions and fermion pair production in association with a high energy initial state photon radiated at low polar angle.

A short summary of the relevant features of the analyses for the pair- and single-production mechanisms is given in the following two sections. Details on the different selections are discussed elsewhere $[6$, $20]$.

\section{Pair production}

The search for pair-produced charged and neutral excited leptons relies on many selections, aimed to maximise the sensitivity for excited lepton masses close to the kinematic limit, around $\sqrt{s} / 2$. For these mass values, radiative and charged-current decays include over $80 \%$ of the decay modes, for any choice of the couplings. Neutral-current decays are hence not considered. Table 3 summarises the selections used for the analysis of each final state, as described below.

Radiative decays of charged excited leptons are searched for through exclusive final states with a pair of identified leptons and two identified photons.
Table 3

Selections used in the search for pair-produced excited leptons and the corresponding numbers of observed events, $N_{D}$, expected background, $N_{B}$, and selection efficiency, $\varepsilon$, for an excited lepton mass of $101 \mathrm{GeV}$. A tighter cut on the missing energy is applied in the $v^{*} v^{*} \rightarrow v \gamma \ell \mathrm{W}$ selection with respect to the $\ell^{*} \ell^{*} \rightarrow \ell \gamma \nu \mathrm{W}$ one

\begin{tabular}{|c|c|c|c|c|}
\hline \multicolumn{5}{|c|}{ Pair production } \\
\hline Signal & Selection & $N_{D}$ & $N_{B}$ & $\varepsilon(\%)$ \\
\hline $\mathrm{e}^{*} \mathrm{e}^{*} \rightarrow$ ee $\gamma \gamma$ & Leptons & 0 & 0.1 & 45 \\
\hline$\mu^{*} \mu^{*} \rightarrow \mu \mu \gamma \gamma$ & and & 0 & 0.3 & 44 \\
\hline$\tau^{*} \tau^{*} \rightarrow \tau \tau \gamma \gamma$ & photons & 0 & 0.4 & 40 \\
\hline$\ell^{*} \ell^{*} \rightarrow \nu \nu \mathrm{WW}$ & qqqq or qq $\ell v$ & 3012 & 2974 & 66 \\
\hline $\mathrm{e}^{*} \mathrm{e}^{*} \rightarrow \mathrm{e} \gamma \nu \mathrm{W}$ & $\mathrm{e}+\gamma+\not+[\mathrm{qq}$ or $\ell]$ & 9 & 10 & 56 \\
\hline$\mu^{*} \mu^{*} \rightarrow \mu \gamma \nu \mathrm{W}$ & $\mu+\gamma+\not E+[$ qq or $\ell]$ & 6 & 5 & 49 \\
\hline$\tau^{*} \tau^{*} \rightarrow \tau \gamma \nu \mathrm{W}$ & jet $+\gamma+\not k+[$ qq or $\ell]$ & 45 & 43 & 35 \\
\hline$v^{*} \nu^{*} \rightarrow \nu v \gamma \gamma$ & $\gamma \gamma+\not$ & 2 & 1.9 & 45 \\
\hline$v_{\mathrm{e}}^{*} v_{\mathrm{e}}^{*} \rightarrow$ eeWW & $\mathrm{ee}+[\mathrm{qqqq}$ or qq $\ell \nu]$ & 1 & 0.2 & 15 \\
\hline$v_{\mu}^{*} v_{\mu}^{*} \rightarrow \mu \mu \mathrm{WW}$ & $\mu \mu+[\mathrm{qqqq}$ or $\mathrm{qq} \ell \nu]$ & 2 & 0.6 & 19 \\
\hline$\nu_{\tau}^{*} \nu_{\tau}^{*} \rightarrow \tau \tau \mathrm{WW}$ & qqqq or qq $\ell v$ & 3012 & 2974 & 70 \\
\hline$v_{\mathrm{e}}^{*} v_{\mathrm{e}}^{*} \rightarrow v \gamma \mathrm{eW}$ & $\mathrm{e}+\gamma+\not+[\mathrm{qq}$ or $\ell]$ & 10 & 11 & 35 \\
\hline$v_{\mu}^{*} v_{\mu}^{*} \rightarrow v \gamma \mu \mathrm{W}$ & $\mu+\gamma+\not k+[$ qq or $\ell]$ & 10 & 11 & 29 \\
\hline$v_{\tau}^{*} \nu_{\tau}^{*} \rightarrow v \gamma \tau \mathrm{W}$ & jet $+\gamma+\not k+[$ qq or $\ell]$ & 10 & 11 & 23 \\
\hline
\end{tabular}

Final states with two photons and missing energy and momentum are used to tag radiative decays of excited neutrinos.

Four selections are devised to identify the pair of $\mathrm{W}$ bosons produced by the charged-current decays of both charged and neutral excited leptons. Three selections, collectively denoted as qq $\ell v$, and different according to the lepton flavour, select final states with two hadronic jets and an isolated high energy lepton. The fourth selection, qqqq, identifies high multiplicity final states, with four hadronic jets. The qq $\ell v$ and qqqq selections are used for the charged-current decays of charged excited leptons and tau excited neutrinos. Fig. 1a shows the hadronic mass for events selected by the qq $\ell v$ selection. In Fig. $1 \mathrm{~b}$ is shown the sum of the invariant and recoil masses for two jets associated to a reconstructed $\mathrm{W}$ in the qqqq selection. In the search for electron and muon excited neutrinos, an additional pair of electrons and muons is required in addition to the qq $\ell v$ and qqqq selections.

Events where one excited lepton decays radiatively and the other through the charged-current are investigated with a similar selection for both charged and neutral excited leptons. Events are selected that have an isolated lepton, a photon and missing energy. In addition, the decay products of a $\mathrm{W}$ boson are required 

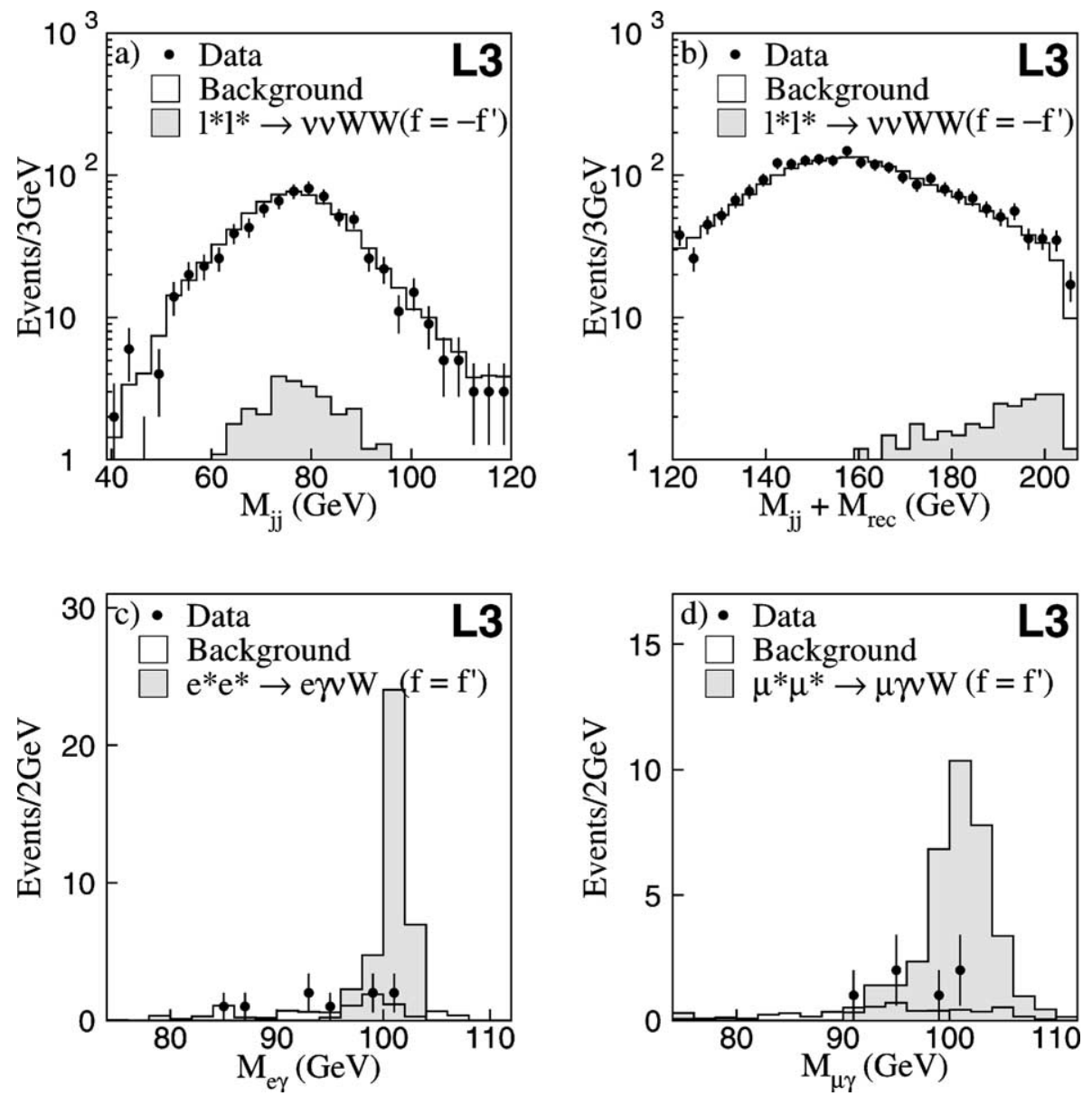

Fig. 1. Distributions of (a) the hadronic invariant mass, $M_{j j}$, in the qq $\ell v$ selection; (b) the sum of $M_{j j}$ and the recoil mass, $M_{\text {rec}}$, for two jets in the qqqq selection; (c) the electron photon invariant mass in the e $\gamma \nu \mathrm{W}$ selection and (d) the muon photon invariant mass in the $\mu \gamma \nu \mathrm{W}$ selection. The expected signal for excited leptons produced in pairs with a mass of $101 \mathrm{GeV}$ is shown together with data and Standard Model background, which is dominated by charged-current four-fermion production in (a) and (b) and by fermion pair-production with initial state radiation in (c) and (d).

as either two hadronic jets or an additional isolated lepton. Tighter requirements on the amount of missing energy are applied for the case of neutral excited leptons. Figs. 1c and d show the masses of the excited electron and muon candidates, reconstructed as the photon-lepton invariant mass.

\section{Single production}

The search for singly produced excited leptons complements the search through the pair-production mechanism and gives access to the mass range from $\sqrt{s} / 2$ up to $\sqrt{s}$. To retain the highest efficiency, all decay modes are investigated: radiative, charged-current and neutral-current. Table 4 lists the association between all final states and the corresponding selections which are summarised below.

In the search for charged excited leptons decaying radiatively, final states with two leptons and a photon are selected. High sensitivity to the excited lepton mass is achieved via the invariant mass of a detected lepton and the photon, as presented in Figs. 2a, $\mathrm{b}$ and $\mathrm{c}$. Events with one photon and large missing energy and momentum are used to search for the possible production of excited neutrinos. Fig. $2 d$ presents 
Table 4

Selections used in the search for singly-produced excited leptons and corresponding numbers of observed events, $N_{D}$, expected background, $N_{B}$, and selection efficiency, $\varepsilon$, for excited lepton masses of 110, 160 and $201 \mathrm{GeV}$. The $\ell \ell \notin$ selections used for the neutral-current decays are tighter than those used for the charged-current decays

\begin{tabular}{|c|c|c|c|c|}
\hline \multicolumn{5}{|c|}{ Single production } \\
\hline Signal & Selection & $N_{D}$ & $N_{B}$ & $\varepsilon(\%)$ \\
\hline $\mathrm{ee}^{*} \rightarrow \mathrm{ee} \gamma$ & Leptons & 672 & 737 & $54,65,65$ \\
\hline$\mu \mu^{*} \rightarrow \mu \mu \gamma$ & and a & 55 & 66 & $62,63,60$ \\
\hline$\tau \tau^{*} \rightarrow \tau \tau \gamma$ & photon & 48 & 60 & $42,45,43$ \\
\hline $\mathrm{ee}^{*} \rightarrow \mathrm{ev}_{\mathrm{e}} \mathrm{W}$ & $\mathrm{qq} \ell v$ or & 749 & 773 & $65,64,61$ \\
\hline$\mu \mu^{*} \rightarrow \mu v_{\mu} \mathrm{W}$ & $\mathrm{qq} \notin$ or & 652 & 675 & $60,64,62$ \\
\hline$\tau \tau^{*} \rightarrow \tau \nu_{\tau} \mathrm{W}$ & $\ell \ell \notin$ & 1287 & 1307 & $59,48,50$ \\
\hline $\mathrm{ee}^{*} \rightarrow \mathrm{eeZ}$ & $\mathrm{qq} \ell v$ or & 673 & 698 & $65,37,63$ \\
\hline$\mu \mu^{*} \rightarrow \mu \mu \mathrm{Z}$ & $\mathrm{qq} \notin$ or & 568 & 596 & $58,41,59$ \\
\hline$\tau \tau^{*} \rightarrow \tau \tau \mathrm{Z}$ & $\ell \ell \notin$ & 1217 & 1236 & $32,22,37$ \\
\hline$v v^{*} \rightarrow v v \gamma$ & $\gamma+\not$ & 176 & 194 & $60,67,70$ \\
\hline$v_{\mathrm{e}} v_{\mathrm{e}}^{*} \rightarrow v_{\mathrm{e}} \mathrm{eW}$ & $\mathrm{qq} \ell v$ or & 749 & 773 & $61,68,67$ \\
\hline$v_{\mu} v_{\mu}^{*} \rightarrow v_{\mu} \mu \mathrm{W}$ & $\mathrm{qq} \notin$ or & 652 & 675 & $63,63,60$ \\
\hline$v_{\tau} v_{\tau}^{*} \rightarrow v_{\tau} \tau \mathrm{W}$ & $\ell \ell \notin$ & 1287 & 1307 & $46,57,60$ \\
\hline$v v^{*} \rightarrow v v Z$ & $\mathrm{qq} \notin$ or $\ell \ell \notin$ & 343 & 350 & $18,15,40$ \\
\hline
\end{tabular}

the normalised energy spectrum of the selected photons.

For the charged-current decay of charged and neutral excited leptons, the qq $\ell v$ selection of the pair-production searches is used. Fig. 3 presents the distribution used for the reconstruction of the mass of the excited lepton candidates, namely the lepton recoil mass in the case of charged excited leptons and the invariant mass of the lepton and the detected jets in the case of excited neutrinos with a hadronic decay of the $\mathrm{W}$ boson. To retain the highest efficiency, two additional selections are devised. They identify events with hadronic activity and missing energy, $\mathrm{qq} \not \mathcal{E}$, as well as leptons and missing energy, $\ell \ell \not E$. These selections complement the qq $\ell v$ selection in the case in which the lepton is not detected or the $\mathrm{W}$ boson decays into leptons.

Neutral-current decays of charged excited leptons are searched for with the same selections used for the charged-current case, supplementing the $\ell \ell \notin$ by the requirement that the visible mass of the two leptons is close to the $Z$ boson mass The neutral-current decay of excited neutrinos produces a $\mathrm{Z}$ boson and missing energy, searched for with the qq $\notin$ and $\ell \ell \notin$ selections. The latter requires a visible mass of the two leptons close to the mass of the $\mathrm{Z}$ boson.

\section{Results}

Tables 3 and 4 list the number of events observed in the data by each selection together with the Monte Carlo background expectations and the signal efficiencies. No evidence for the production of excited leptons is observed in any final state.

Systematic uncertainties affect the results in Tables 3 and 4 . An uncertainty of $1.5-2.5 \%$, depending on the selection, is associated to the background estimate. This includes the uncertainties on the cross sections of background processes, limited Monte Carlo statistics, detector simulation and the selection procedure. The limited Monte Carlo statistics and the detector simulation also affect the estimation of the signal efficiency. Depending on the selection, the systematic uncertainty from this source is around $2.5 \%$, which also covers the accuracy of the efficiency interpolation for different excited lepton masses.

The absence of excited leptons in the data sample is expressed by means of upper limits on their masses and couplings. In the derivation of these limits, the data discussed in this Letter are considered at their luminosity averaged $\sqrt{s}$ of $206 \mathrm{GeV}$. Absolute limits on the masses of charged and neutral excited leptons are derived from the pair-production process, whereas 

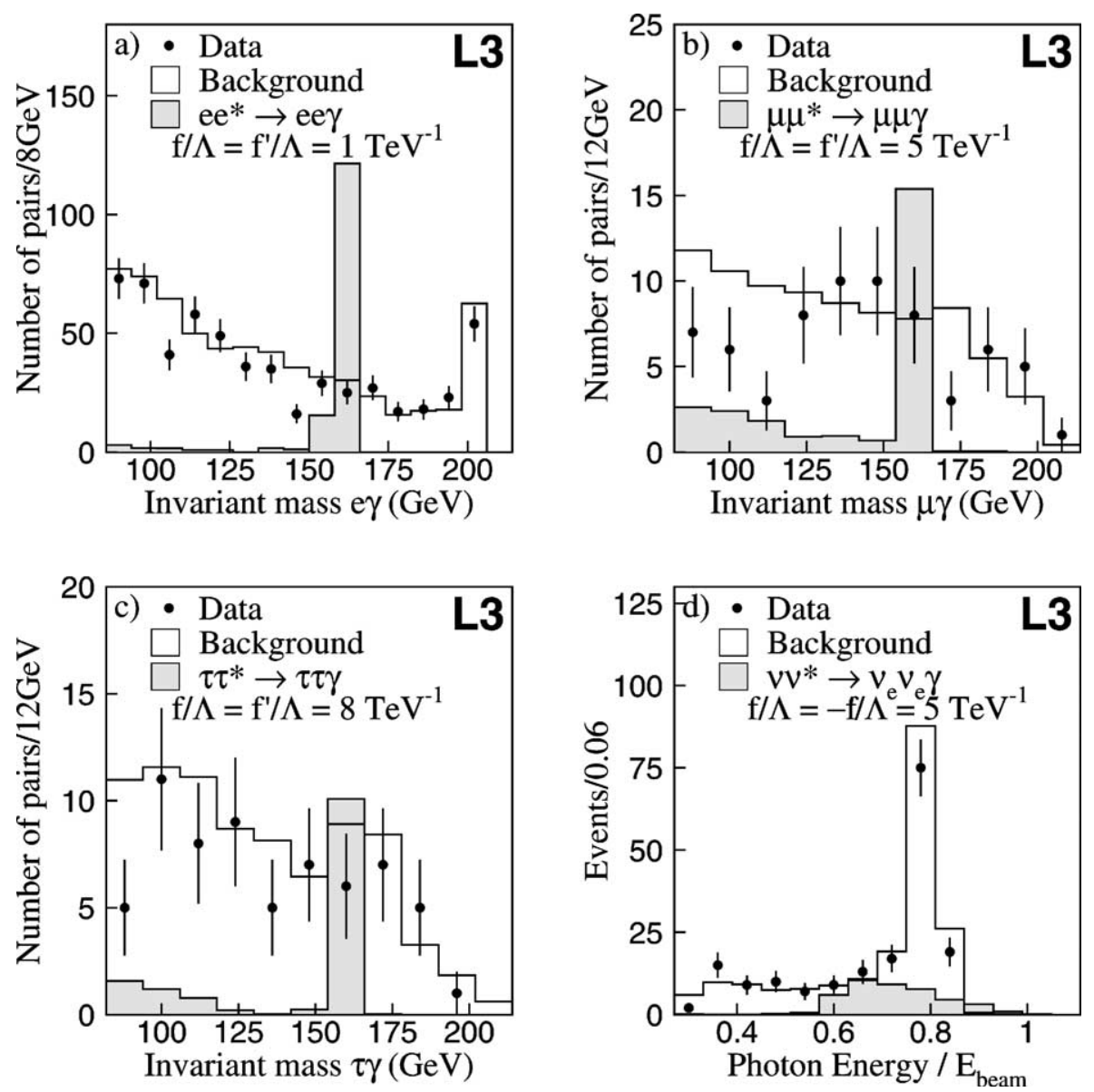

Fig. 2. The invariant mass distributions for the (a) $\mathrm{e}-\gamma$, (b) $\mu-\gamma$, and (c) $\tau-\gamma$ systems. (d) The normalised energy distribution of single photon events. The expected signal for a singly-produced excited lepton with a mass of $160 \mathrm{GeV}$ is shown together with data and the Standard Model background expectation, due to fermion pair-production with initial state radiation photons. The signals are plotted for the arbitrary choice of couplings displayed in the figures.

limits on the effective couplings as a function of the masses are derived from the single-production study. The limits discussed in the following also include the results from previous searches at $\sqrt{s}=$ $189 \mathrm{GeV}$ [5] and $\sqrt{s}=192-202 \mathrm{GeV}$ [6]. All limits take systematic uncertainties into account and are reported for the two scenarios, $f=f^{\prime}$ and $f=$ $-f^{\prime}$, which reflect the different dominant branching ratio and consequent final state topologies. For the pair-production process, the numbers of observed and expected events, together with the signal efficiencies are translated into upper limits on the cross section for the production of excited leptons. A scan is performed for all possible values of the ratio $f / f^{\prime}$, and the decay fractions of charged excited leptons and excited neutrinos are computed for the radiative, chargedcurrent and mixed decay modes. Signal cross sections are calculated and the corresponding mass limits are derived, as presented in Table 5. More stringent limits are obtained for channels with low background. As an example, the limits on charged excited leptons in the $f=f^{\prime}$ scenario and excited neutrinos in the $f=-f^{\prime}$ scenario benefit from a large branching ratio in the clean radiative decay channel. In addition to those corresponding to the $f=f^{\prime}$ and $f=-f^{\prime}$ scenarios, limits are also given for all excited leptons which 

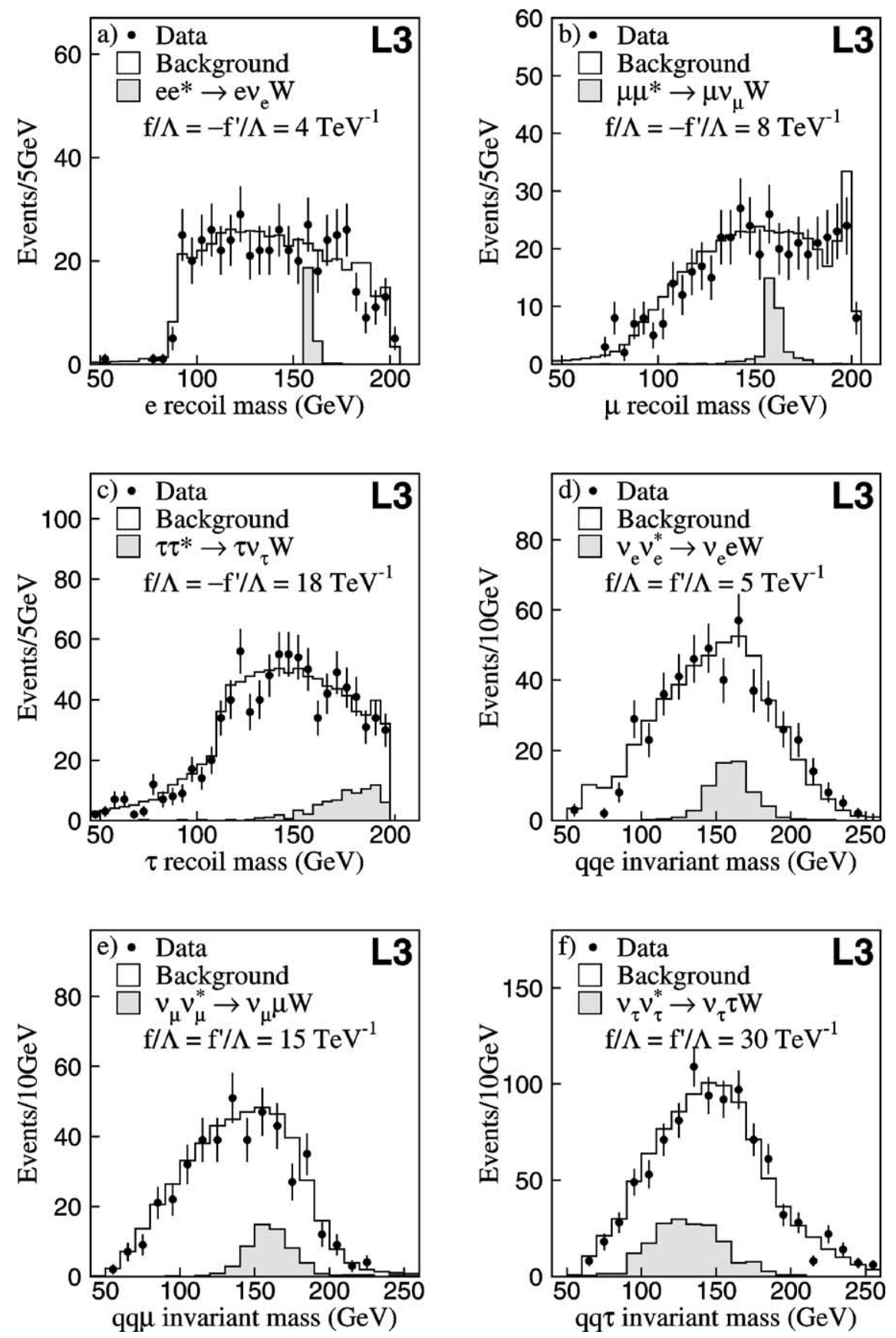

Fig. 3. Lepton recoil mass distributions for the (a) qqev, (b) qq $\mu \nu$ and (c) qq $\tau v$ selections. Invariant mass distributions for the (d) qqev, (e) qq $\mu \nu$ and (f) qq $\tau \nu$ selections. The expected signal for a singly-produced excited lepton with a mass of $160 \mathrm{GeV}$ is shown together with data and Standard Model background expectations dominated by charged-current four-fermion production. The signals are plotted for the arbitrary choice of couplings displayed in the figures. 

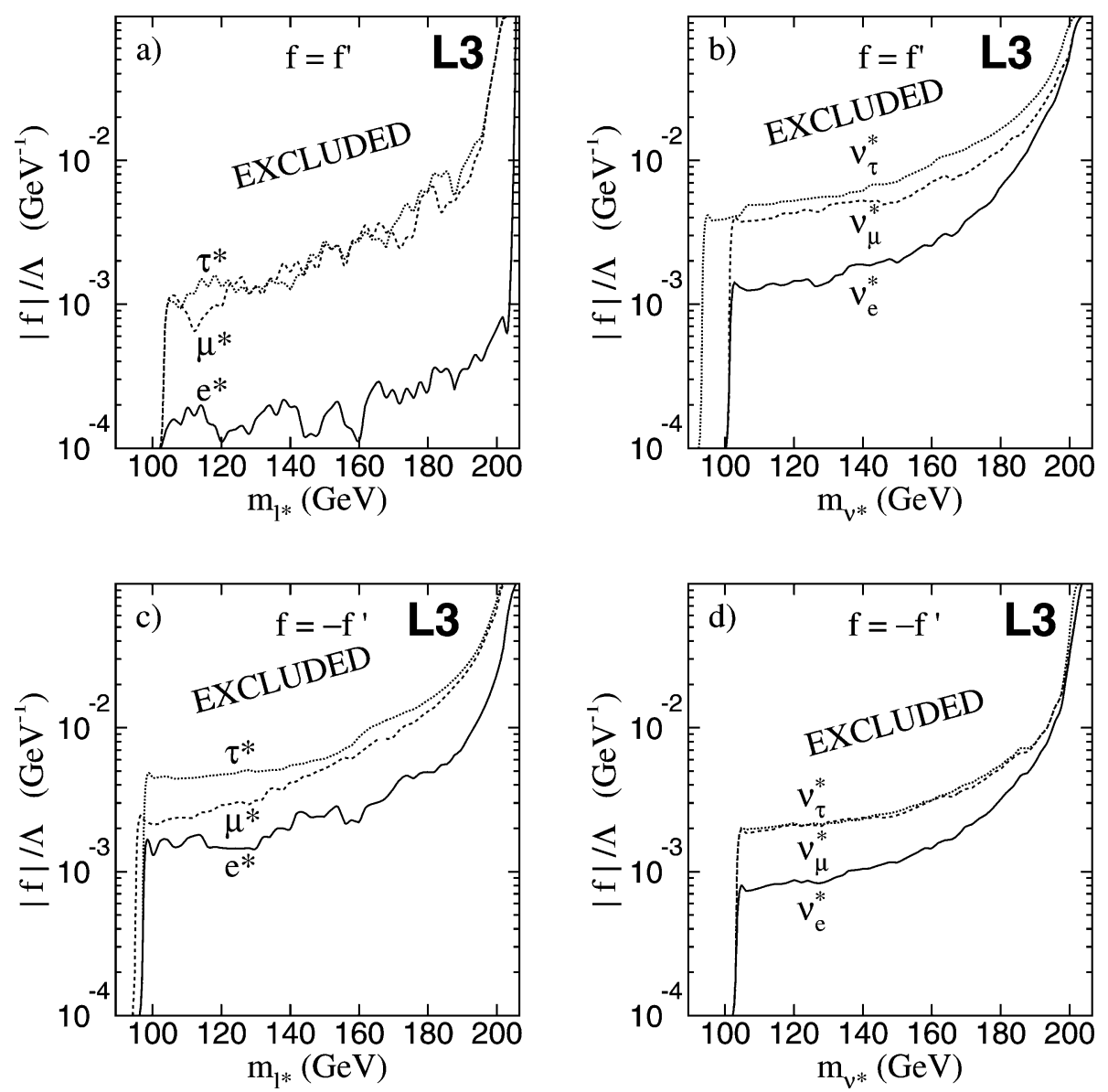

Fig. 4. $95 \%$ confidence level upper limits on $|f| / \Lambda$, as a function of the excited lepton mass with $f=f^{\prime}$ for (a) $\mathrm{e}^{*}, \mu^{*}$ and $\tau^{*}$, (b) $\nu_{\mathrm{e}}^{*}, v_{\mu}^{*}$ and $\nu_{\tau}^{*}$, and with $f=-f^{\prime}$ for (c) $\mathrm{e}^{*}, \mu^{*}$ and $\tau^{*}$, (d) $v_{\mathrm{e}}^{*}, v_{\mu}^{*}$ and $v_{\tau}^{*}$.

Table 5

Lower mass limits at $95 \%$ confidence level for charged and neutral excited lepton as obtained from pair-production searches

\begin{tabular}{cccc}
\hline Excited lepton & \multicolumn{3}{c}{$95 \%$ CL mass limit (GeV) } \\
\cline { 2 - 4 } & $f=f^{\prime}$ & $f=-f^{\prime}$ & Any coupling \\
\hline $\mathrm{e}^{*}$ & 102.8 & 96.6 & 96.5 \\
$\mu^{*}$ & 102.8 & 96.6 & 96.6 \\
$\tau^{*}$ & 102.8 & 96.6 & 95.6 \\
$\nu_{\mathrm{e}}^{*}$ & 101.7 & 102.6 & 101.5 \\
$\nu_{\mu}^{*}$ & 101.8 & 102.6 & 101.4 \\
$\nu_{\tau}^{*}$ & 92.9 & 102.6 & 91.3 \\
\hline
\end{tabular}

correspond to the lowest values obtained in the scan over $f / f^{\prime}$, and hence valid for any choice of the couplings.
In the case of single-production searches, an upper limit on the cross section is obtained as a function of the excited lepton mass. Different mass values are investigated by means of the distributions of the variables presented in Figs. 2 and 3. A linear interpolation of the detection efficiencies as a function of the excited lepton mass is used. These cross section limits are translated in the upper limits on the ratios $|f| / \Lambda$ and $\left|f^{\prime}\right| / \Lambda$ shown in

Fig. 4. The edge of the curves at low mass indicates the lower mass limit derived from pair-production searches, whereas the rise at high mass reflects the decrease of the expected signal cross section and therefore of the experimental sensitivity. The limits corresponding to charged excited leptons in the $f=f^{\prime}$ scenario and excited neutrinos in the $f=-f^{\prime}$ scenario 
are derived mainly from the radiative decay searches, whose clean final states have a high signal sensitivity. These limits are more stringent than those obtained in the complementary scenarios in which the radiative decays are forbidden. The limits corresponding to excited leptons of the first generation are significantly tighter due to their higher cross section resulting from the $t$-channel contribution.

In conclusion, no evidence for charged and neutral excited leptons of any flavour is found in the LEP data, and lower mass limits as high as $101.5 \mathrm{GeV}$ are derived for any value of the excited lepton couplings. Upper limits on $|f| / \Lambda$ and $\left|f^{\prime}\right| / \Lambda$, ranging from $10^{-1}$ to $10^{-4} \mathrm{GeV}^{-1}$ according to the excited lepton flavour and mass, are set in the mass range from 100 to $200 \mathrm{GeV}$.

\section{References}

[1] K. Hagiwara, et al., Z. Phys. C 29 (1985) 115.

[2] M.B. Voloshin, et al., Sov. Phys. JETP 64 (1986) 446; M.B. Voloshin, Phys. Lett. B 209 (1988) 360; F. Boudjema, et al., Phys. Lett. B 240 (1990) 485; F. Boudjema, et al., Z. Phys. C 57 (1993) 425.

[3] F. Boudjema, et al., in: J. Ellis, et al. (Eds.), Z. Physics at LEP 1, Vol. 2, 1989, p. 188, Report CERN/89/08, and references therein.

[4] S.L. Glashow, Nucl. Phys. 22 (1961) 579; A. Salam, in: N. Svartholm (Ed.), Elementary Particle Theory, Almqvist-Wiksell, Stockholm, 1968;

S. Weinberg, Phys. Rev. Lett. 19 (1967) 1264.

[5] L3 Collaboration, M. Acciarri, et al., Phys. Lett. B 473 (2000) 177.

[6] L3 Collaboration, M. Acciarri, et al., Phys. Lett. B 502 (2001) 37.

[7] ALEPH Collaboration, D. Buskulic, et al., Phys. Lett. B 385 (1996) 445 ; DELPHI Collaboration, P. Abreu, et al., E. Phys. J. C 8 (1999) 41 ;

OPAL Collaboration, G. Abbiendi, et al., Eur. Phys. J. C 14 (2000) 73;
OPAL Collaboration, G. Abbiendi, et al., Phys. Lett. B 544 (2002) 57.

[8] H1 Collaboration, C. Adloff, Phys. Lett. B 525 (2002) 9; H1 Collaboration, C. Adloff, Phys. Lett. B 548 (2002) 35; ZEUS Collaboration, S. Chekanov, et al., Phys. Lett. B 549 (2002) 32.

[9] L3 Collaboration, B. Adeva, et al., Nucl. Instrum. Methods A 289 (1990) 35;

L3 Collaboration, O. Adriani, et al., Phys. Rep. 236 (1993) 1; M. Chemarin, et al., Nucl. Instrum. Methods A 349 (1994) 345; M. Acciarri, et al., Nucl. Instrum. Methods A 351 (1994) 300; G. Basti, et al., Nucl. Instrum. Methods A 374 (1996) 293;

A. Adam, et al., Nucl. Instrum. Methods A 383 (1996) 342.

[10] BHWIDE version 1.01 is used; S. Jadach, et al., Phys. Rev. D 40 (1989) 3582;

S. Jadach, et al., Comput. Phys. Commun. 70 (1992) 305;

S. Jadach, et al., Phys. Lett. B 390 (1997) 298.

[11] TEEGG Monte Carlo; D. Karlen, Nucl. Phys. B 289 (1987) 23.

[12] KK2F version 4.12 is used; S. Jadach, et al., Comput. Phys. Commun. 130 (2000) 260.

[13] KORALW version 1.33 is used; S. Jadach, et al., Comput. Phys. Commun. 94 (1996) 216; M. Jadach, et al., Phys. Lett. B 372 (1996) 289.

[14] EXCALIBUR Monte Carlo; F.A. Berends, et al., Comput. Phys. Commun. 85 (1995) 437.

[15] PYTHIA versions 5.722 and 6.1 are used; T. Sjöstrand, Preprint CERN-TH/7112/93 (1993), revised August 1995;

T. Sjöstrand, Comput. Phys. Commun. 82 (1994) 74; T. Sjöstrand, hep-ph/0001032.

[16] GGG Monte Carlo; F.A. Berends, et al., Nucl. Phys. B 186 (1981) 22; CALKUL Collaboration, F.A. Berends, et al., Nucl. Phys. B 239 (1984) 395.

[17] PHOJET version 1.05 is used; R. Engel, Z. Phys. C 66 (1995) 203; R. Engel, et al., Phys. Rev. D 54 (1996) 4244.

[18] DIAG36 Monte Carlo; F.A. Berends, et al., Nucl. Phys. B 253 (1985) 441.

[19] GEANT version 3.15 is used; R. Brun et al., Preprint CERN-DD/EE/84-1 (1984), revised 1987;

The GHEISHA program (H. Fesefeldt, RWTH Aachen Report PITHA 85/02 (1985)) is used to simulate hadronic interactions.

[20] M. Falagan, Ph.D. thesis, Complutense University, Madrid, 2000. 